\title{
An Automated Approach to Connectivity-Based Partitioning of Brain Structures
}

\author{
P.A. Cook ${ }^{1}$, H. Zhang ${ }^{2}$, B.B. Avants ${ }^{2}$, P. Yushkevich ${ }^{2}$, D.C. Alexander ${ }^{1}$, \\ J.C. Gee ${ }^{2}$, O. Ciccarelli ${ }^{3}$, and A.J. Thompson ${ }^{3}$ \\ 1 Centre for Medical Image Computing, Department of Computer Science, \\ University College London, UK \\ 2 Departments of Computer \& Information Science and Radiology, \\ University of Pennsylvania, Philadelphia PA 19104, USA \\ 3 Department of Headache, Brain Injury and Neuroinflammation, \\ Institute of Neurology, University College London, UK
}

\begin{abstract}
We present an automated approach to the problem of connectivity-based partitioning of brain structures using diffusion imaging. White-matter fibres connect different areas of the brain, allowing them to interact with each other. Diffusion-tensor MRI measures the orientation of white-matter fibres in vivo, allowing us to perform connectivity-based partitioning non-invasively. Our new approach leverages atlas-based segmentation to automate anatomical labeling of the cortex. White-matter connectivities are inferred using a probabilistic tractography algorithm that models crossing pathways explicitly. The method is demonstrated with the partitioning of the corpus callosum of eight healthy subjects.
\end{abstract}

\section{Introduction}

Diffusion-weighted MRI (DW-MRI) offers insight into the structure of whitematter fibres in the brain. From DW-MRI we can infer the local fibre orientation [1], and "tractography" algorithms use this information to reconstruct entire fibre pathways [2] The possibility to parcellate brain structures based on their anatomical connectivity has important implications since it may help to identify functionally distinct subregions of the brain [3, 4]. Correspondence between anatomical connectivity and functional activation has been shown in the thalamus [5].

In this paper, we present an automated approach to brain region parcellation based on connectivity to cortical grey-matter inferred from fibre tractography. Compared to earlier efforts [3, 4, 6, our work's original contributions can be outlined as follows. Firstly, we leverage advances in atlas-based image segmentation to produce cortex parcellation of subjects automatically. Secondly, we resolve crossing fibres and use a novel model of uncertainty when two fibre bundles are present within a voxel. We begin by reviewing the basic concepts of diffusion imaging, tractography and connectivity-based parcellation. We then present methods and results of our approach, followed by a discussion of future works. 


\subsection{Diffusion-Tensor MRI}

In diffusion-tensor MRI (DT-MRI) [7, we fit a Gaussian model of the displacement density

$$
p(\mathbf{r}, t)=G(\mathbf{r} ; \mathrm{D}, t)=\left((4 t)^{3} \operatorname{det}(\mathrm{D})\right)^{-\frac{1}{2}} \exp \left(-\mathbf{r}^{\mathrm{T}} \mathrm{D}^{-1} \mathbf{r}(4 t)^{-1}\right)
$$

of diffusing water molecules after time $t$, where $\mathbf{r}$ is the displacement and $\mathrm{D}$ is a second-order symmetric tensor, with eigenvalues $\lambda_{1} \geq \lambda_{2} \geq \lambda_{3}$ and corresponding eigenvectors $\mathbf{e}_{1}, \mathbf{e}_{2}$ and $\mathbf{e}_{3}$. A useful statistic from D is the fractional anisotropy [8], which quantifies the anisotropy in the diffusion with a value between 0 (isotropic diffusion) and 1 (displacement is nonzero only along $\mathbf{e}_{1}$ ).

In voxels containing a single bundle of axonal fibres with a common orientation, $\lambda_{1} \gg \lambda_{2} \simeq \lambda_{3}$, and the principal direction $\mathbf{e}_{1}$ provides an estimate of the direction of the fibre bundle. However, when the tissue structure in the voxel is more complex, such as at fibre crossings, the Gaussian model is a poor approximation of $p$, and $\mathbf{e}_{1}$ is not a reliable indicator of the fibre orientation. A variety of techniques exist to resolve the orientations of crossing fibres [9].

\subsection{Tractography}

Diffusion-tensor MRI provides estimates of the local fibre orientation in each voxel, which typically occupies a volume on the order of $10^{-9} \mathrm{~m}^{3}$. Tractography uses the local fibre-orientation measurements to reconstruct entire axonal paths.

Simple tractography calculates streamlines, which follow the local fibre-orientation estimate from voxel to voxel. Streamline trajectories are sensitive to noise, and authors often reduce false-positive connections by imposing limits on the streamline curvature, which can be as low as 45 degrees, and by specifying a minimum anisotropy for tracking to continue, since the fibre orientation is not well defined at low anisotropy [10.

Monte-Carlo based probabilistic methods, such as those proposed by Behrens et al [6] and Parker et al [1], define a probability density function (PDF) on the true fibre orientation in each voxel from the DW-MRI data. Streamlines are tracked repeatedly from a single seed point. Each time, fibre orientations are drawn from the PDF in each voxel. Over a large number of iterations, the process yields a connection probability from the seed point to any other voxel $v$, which is the fraction of streamlines that pass through $v$. The PDF is less concentrated when there is high uncertainty in the fibre orientation, for example in voxels with low anisotropy. This means that the set of streamlines passing through regions of high uncertainty disperse rapidly, which results in lower connection probability. Since probabilistic algorithms give a measurement of the confidence in each potential connection, they do not require restrictive thresholds.

\subsection{Connectivity-Based Parcellation of the Brain}

Tractography, combined with anatomic labeling of cortex, makes possible the non-invasive study of anatomical connectivity between distinct cortical structures. Connectivity-based parcellation is a method for segmenting a region of 
interest in the brain based on anatomical connectivity to other brain regions. Given a segmentation of the brain into distinct labelled regions, the method uses tractography to determine which labelled region is most likely to be connected to each voxel in the region of interest.

The method can be performed using either deterministic or probabilistic algorithms. However, the anisotropy threshold in deterministic algorithms limits their ability to identify pathways near to grey-matter areas, such as cortex, and the tight curvature restriction penalizes genuine pathways demonstrating high curvature. Although such algorithms have been applied to parcellate the corpus callosum [3, 4], their known limitations require extra care in interpreting the results they produce.

Behrens et al [6] use a probabilistic approach to partition the thalamus, a deep grey-matter structure. Behrens uses a Bayesian method to define a single-fibre PDF. The cortex is segmented into several functional zones, and probabilistic streamlines are tracked from seed points in the thalamus. The cortical zone with the highest connection probability to the seed point is the zone that contains the most probabilistic streamlines. The thalamic segmentation is consistent with previous postmortem histological studies of connectivity.

\section{Method}

We will refer to the brain region of interest as the seed region and each voxel in the seed region as a seed point. We define the "connectivity map" for each seed point as the collection of probabilistic streamlines emanating from the seed.

The algorithm to partition the seed region is as follows:

1. Label the grey-matter regions of the subject in the space of the diffusion data.

2. Generate the connectivity map for each seed point from the diffusion data.

3. For each seed point, determine the labelled grey-matter region with the highest connection probability.

Steps 1 and 2 of the algorithm are described in detail below. In step 3, we use the same approach as Behrens et al, which we described in section 1.3 .

\subsection{Cortical Region Labeling}

We require cortical regions to be defined in the same space as the connectivity maps. However the diffusion-weighted images lack the resolution for accurate delineation of cortex. Thus a high resolution T1-weighted image of the subject is acquired, on which the cortical regions of interest are defined. These regions are then transferred onto the diffusion data after co-registration of the T1-weighted to a non-diffusion-weighted image acquired as part of the diffusion MRI sequence, hereafter refered to as the $[b=0]$ image.

One feature of our method is the automation of the cortical region labeling step by warping an atlas into alignment with the T1-weighted image. Figure 1depicts the brain atlas and its warped version after registration to the T1-weighted 

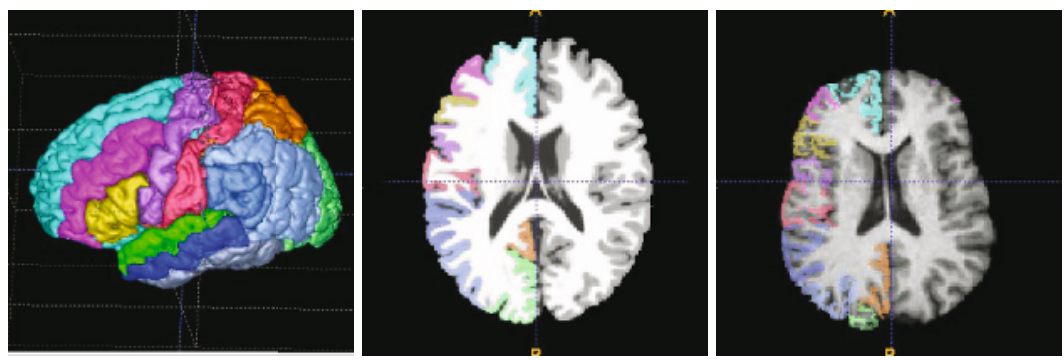

Fig. 1. Atlas-based brain image segmentation. (Left) A surface rendering of the labeled atlas used in this work. (Middle) The grey-matter labels for one hemisphere are shown superimposed on the underlying structural image of the atlas. (Right) The atlas is registered to the T1-weighted image of one subject, and the warped grey-matter labels for one hemisphere are shown superimposed on the subject's structural image. The partition results of the subject's corpus callosum is shown in figure 2 which also contains the color-coded list of the cortical regions delineated in the atlas, further details of which can be found in 12 .

image of one subject. To improve the quality of the co-registration between the T1-weighted and $[b=0]$ images, we also acquire a high-resolution EPI image, which is used as an intermediate representation of the brain configuration in the diffusion data space. Specifically, the T1-weighted image is non-rigidly aligned to the EPI image, which in turn is non-rigidly registered to the $[b=0]$ image. The corresponding transformations are computed to enable atlas labels defined on the T1-weighted image to be transferred to the $[b=0]$ image. The non-rigid registration algorithm used optimizes an intensity cross-correlation metric under the constraints of a diffeomorphic transformation model in multiresolution fashion [13.

\subsection{Generation of Probabilistic Streamlines}

Before we generate probabilistic streamlines, we must complete three stages of pre-processing of the DW-MRI data: first classifying voxels as containing zero, one, or multiple fibre bundles, then determining fibre orientations in each voxel, and finally calibrating fibre-orientation PDFs. The first two steps must be completed for each subject, while the last is performed once.

Fibre-Orientation Estimation. In a similar way to Parker and Alexander 14, we use the spherical-harmonic classification and fibre-orientation estimation algorithm of Alexander et al [15] to determine which voxels contain zero, one, or multiple fibre populations. When the algorithm detects multiple fibres, we use a mixture of two zero-mean Gaussian distributions to model $p$, otherwise we use the single Gaussian as in DT-MRI (Eq. 1). We use Levenberg-Marquardt optimization to fit the parameters of the models to the data. We reduce the complexity of the two-fibre model by assuming two Gaussian compartments in 
the voxel, mixed in equal proportion, and that the diffusion tensors are both cylindrically symmetric, so $\lambda_{1} \geq \lambda_{2}=\lambda_{3}$. The principal eigenvector of each tensor provides a separate fibre-orientation estimate.

Calibration of the Fibre-Orientation PDF. In single-tensor voxels, we use an existing PDF proposed by Cook et al [16, based on the Watson distribution [17:

$$
f(\mathbf{x})=W( \pm \mathbf{x} ; \boldsymbol{\mu}, \kappa)=M\left(\frac{1}{2}, \frac{3}{2}, \kappa\right)^{-1} \exp \left(\kappa\left(\boldsymbol{\mu}^{\mathrm{T}} \mathbf{x}\right)^{2}\right),
$$

where $M$ denotes the confluent hypergeometric function of the first kind and $\mathbf{x}$ is a unit axis. The parameter $\kappa$ determines the concentration of samples about the mean axis $\boldsymbol{\mu}$. We construct a lookup table that predicts $\kappa$ from the tensor shape parameters $\frac{\lambda_{1}}{\lambda_{3}}$ and $\frac{\lambda_{2}}{\lambda_{3}}$. These indices are sufficient to fully specify a tensor, because the trace $\lambda_{1}+\lambda_{2}+\lambda_{3}$ is approximately constant in brain tissue. For each entry in the lookup table we synthesize measurements predicted by $p(\mathbf{r})=$ $G(\mathbf{r}, \mathrm{D}, t)$, add complex Gaussian noise and fit the diffusion tensor to the noisy measurements. We repeat this process to obtain a large sample of noisy principal directions, $\mathbf{x}_{i}, 1 \leq i \leq 10,000$, from which we estimate $\kappa$ numerically [17.

In voxels containing two fibre populations, we use a Watson PDF for each fibre bundle. We calibrate the PDF by adding noise to the synthetic signal from $p(\mathbf{r})=0.5\left(G\left(\mathbf{r}, \mathrm{D}_{1}, t\right)+G\left(\mathbf{r}, \mathrm{D}_{2}, t\right)\right)$, but we compute fewer samples than for the single-fibre $\mathrm{PDF}$ because generating two-fibre samples is more computationally expensive. The resulting samples $\mathbf{x}_{i}, 1 \leq i \leq 4,000$ contain both fibre-orientation estimates from each trial. We estimate the concentration of both distributions simultaneously by maximising the log-likelihood $l$ of the axes:

$$
l\left(W_{1}, W_{2}\right)=\sum_{i=1}^{4000} \log \left(\frac{1}{2}\left[W_{1}\left(\mathbf{x}_{i} ; \boldsymbol{\mu}_{1}, \kappa_{1}\right)+W_{2}\left(\mathbf{x}_{i} ; \boldsymbol{\mu}_{2}, \kappa_{2}\right]\right) .\right.
$$

Previously, Parker and Alexander [14 constructed a two-fibre PDF by sorting the samples into two groups and fitting a Gaussian model to each. The objective function in Eq. 3 requires no assignment of axes to a particular fibre, and hence avoids any potential bias from incorrect assignment. We optimize $l\left(W_{1}, W_{2}\right)$ with repeated runs of the Levenberg-Marquardt algorithm. We construct a lookup table that gives $\kappa_{1}$ and $\kappa_{2}$ as a function of the fractional anisotropy of each tensor, and the angle at which the two principal directions cross.

Tractography. We use no anisotropy threshold in the tractography, and we apply a minimal curvature threshold to prevent streamlines from looping back on themselves and inflating the connection probability. We track by following the principal direction in each voxel, without interpolation. In voxels containing two fibres, we make a probabilistic choice of whether to sample from $W_{1}$ or $W_{2}$ based on the previous direction of the streamline, $\mathbf{x}_{p}$. The probability of sampling $W_{1}$ is $p_{1}=W_{1}\left(\mathbf{x}_{p} ; \boldsymbol{\mu}_{1}, \kappa_{1}\right)\left[W_{1}\left(\mathbf{x}_{p} ; \boldsymbol{\mu}_{1}, \kappa_{1}\right)+W_{2}\left(\mathbf{x}_{p} ; \boldsymbol{\mu}_{2}, \kappa_{2}\right)\right]^{-1}$, and the probability of choosing $W_{2}$ is $\left(1-p_{1}\right)$. We track fibres from the seed points in both directions, 
and we stop tracking if the streamline either curves by more than $80^{\circ}$ over the length of one voxel, reaches the brain surface, or intersects itself.

\section{Results and Discussions}

We demonstrate the method by partitioning the corpus callosum (CC), the structure through which the two cerebral hemispheres communicate, using the brain images from 8 healthy volunteers. The diffusion imaging sequence uses 60 diffusion weighted measurements in each voxel. The first 54 measurements are at a fixed $|\mathbf{q}|$ giving $b=1050 \mathrm{smm}^{-2}$, with the gradient directions spread evenly on the hemisphere to minimise dependence of the tensor upon the orientation of the tissue relative to the gradient directions. Six of the 54 diffusion-weighted measurements are repeated with $b=260 \mathrm{smm}^{-2}$, and six measurements are made with $b=0$. We estimate the signal to noise ratio in each $[b=0]$ image to be approximately 14 in white-matter.

Because the fibre bundles originating from the $\mathrm{CC}$ extend to both the left and right cortex, we construct two connectivity maps for each seed point. Each map represents the connections to one side of the brain and is used to derive the partitioning for the corresponding hemisphere. An example of the partitioning is shown in figure 2 ,

A useful statistic is the relative sizes of the partitions which are computed for all subjects and shown in fig. 2. Evidently there are four primary partitions in the CC. Although this is consistent with the earlier study [3], in our study the cortical connectivity is better localized because we have chosen a significantly finer cortical labeling. The voxels within these partitions are most likely to connect to the superior frontal gyrus, the superior parietal gyrus, the occipital gyrus and the parahippocampal gyrus, respectively. On average, over all the controls, the sizes of the largest partitions found, computed as a percentage of
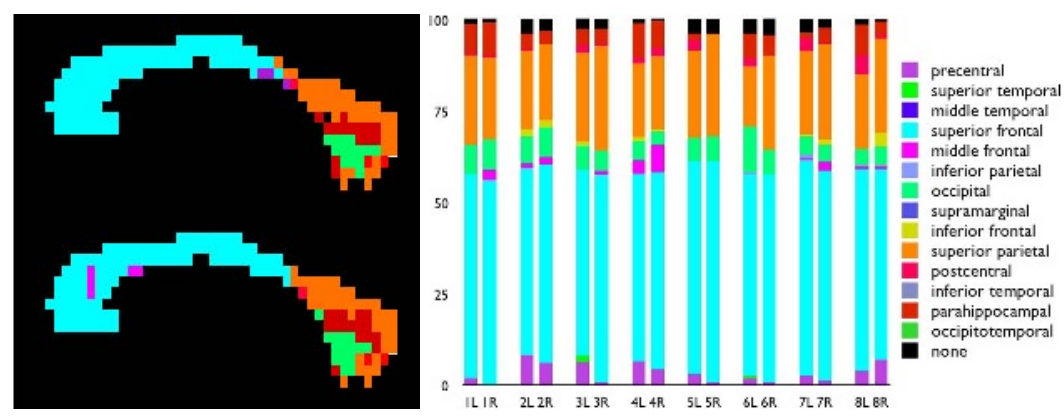

Fig. 2. The partitioning of the corpus callosum. On the left is the partitioning of the subject used in figure 1 for the left (top) and right (bottom) hemispheres. In the centre are the relative sizes of each partition as a percentage of the total area of the corpus callosum, for the left and right connectivity maps from all 8 subjects. On the right is the color-coded list of the cortical regions delineated in the atlas shown in fig. [1 
the size of the corpus callosum, are $53 \%, 22 \%, 7 \%$ and $5 \%$ for the left hemisphere, and are $55 \%, 26 \%, 5 \%$ and $5 \%$ for the right hemisphere. Despite the finer cortical labelling scheme, it appears that the majority of the connections through the corpus callosum are restricted to several large cortical regions. Additionally, several regions, notably the middle and inferior temporal gyrus, the occipitotemporal gyrus and the supramarginal gyrus, are not found in the partitions. It is possible that the connections to these regions are mediated through other regions, either by ipsilateral or U-fiber connections. Equally likely is that other connections exist across the corpus callosum but are more difficult to track reliably using current methods. Certain connections may be relatively difficult to track for several reasons, for example the fibre bundles may be smaller, or the bundles may extend through complex fibre architecture such as fibre crossings.

Also worth noting is the difference in the partitioning results from the left and the right hemispheres. postmortem studies [18] show that there are widespread heterotopic commissure connections across the corpus callosum. Partitioning studies such as this have the potential to offer new evidence of the existence of the heterotopic connections and, furthermore, possibly locate where such connections traverse the corpus callosum.

\section{Conclusion}

We have presented a method for parcellating brain structures based on connectivity maps derived from diffusion MRI data. The method is demonstrated with the partitioning of the corpus callosum. We construct connectivity maps using a novel probabilistic tractography algorithm that resolves crossing fibres. One feature of our method is the automated cortical region labeling based on our atlas-based and registration-driven segmentation technique. Future work includes quantitatively assessing the quality of the automated labeling compared to a manual delineation of the cortex.

\section{Acknowledgement}

PAC is funded by the MIAS IRC, EPSRC GR/N14248/01. OC and AT thank the MS Society of Great Britain and Northern Ireland for their generous support of the NMR Unit, Institute of Neurology. OC is a Wellcome Trust Advanced Fellow. JG thanks the NIH for their generous support through grants NS044189, DA015886 and NS045839. The authors thank Claudia Wheeler-Kingshott (Institute of Neurology, UCL), for the acquisition of the MR images.

\section{References}

1. Pierpaoli, C., Jezzard, P., Basser, P.J., Barnett, A., Chiro, G.D.: Diffusion tensor imaging of the human brain. Radiology 201 (1996) 637-648

2. Mori, S., van Zijl, P.C.M.: Fiber tracking: principles and strategies - a technical review. NMR in Biomed. 15 (2002) 468-480 
3. Huang, H., Zhang, J., Jiang, H., Wakana, S., Poetscher, L., Miller, M.I., van Zijl, P.C.M., Hillis, A.E., Wytike, R., Mori, S.: DTI tractography based parcellation of white matter: Application to the mid-sagittal morphology of corpus callosum. NeuroImage (2004) doi:10.1016/j.neuroimage.2005.01.019

4. Gee, J.C., Zhang, H., Dubb, A., Avants, B.A., Yushkevich, P.A., Duda, J.T.: Anatomy-based visualizations of diffusion tensor images of brain white matter. In Welckert, J., Hagen, H., eds.: Visualization and Image Processing of Tensor Fields. Berlin:Springer (in press)

5. Johansen-Berg, H., Behrens, T.E., Sillery, E., Ciccarelli, O., Thompson, A.J., Smith, S.M., Matthews, P.M.: Functional-anatomical validation and individual variation of diffusion tractography-based segmentation of the human thalamus. Medical Image Analysis 15 (2005) 31:39

6. Behrens, T.E., Johansen-Berg, H., Woolrich, M.W., Smith, S.M., WheelerKingshott, C.A., Boulby, P.A., Barker, G.J., Sillery, E.L., Sheehan, K., Ciccarelli, O., Thompson, A.J., Brady, J.M., Matthews, P.M.: Non-invasive mapping of connections between human thalamus and cortex using diffusion imaging. Nat. Neurosci. 6 (2003) 750-757

7. Basser, P.J., Mattiello, J., Bihan, D.L.: MR diffusion tensor specstroscopy and imaging. Biophys J 66 (1994) 259-267

8. Basser, P.J., Pierpaoli, C.: Microstructural and physiological features of tissues elucidated by quantitative-diffusion-tensor MRI. JMR B111 (1996) 209-219

9. Alexander, D.C.: An introduction to computational diffusion MRI: the diffusion tensor and beyond. In Weichert, J., Hagen, H., eds.: Visualization and Image Processing of Tensor Fields. Springer (2005)

10. Stieltjes, B., Kaufmann, W.E., van Zijl, P.C.M., Fredericksen, K., Pearlson, G.D., Solaiyappan, M., Mori, S.: Diffusion tensor imaging and axonal tracking in the human brainstem. NeuroImage 14 (2001) 723-735

11. Parker, G.J.M., Wheeler-Kingshott, C.A.M., Haroon, H.A.: A framework for a streamline-based probabilistic index of connectivity (PICo) using a structural interpretation of MRI diffusion measurements. JMRI 18 (2003) 242-254

12. Dubb, A., Yushkevich, P.A., Xie, Z., Gur, R.C., Gur, R.E., Gee, J.C.: Regional structural characterization of the brain of schizophrenia patients. In: Proc. MICCAI. (2004)

13. Avants, B.A., Schoenemann, P.T., Gee, J.C.: Lagrangian frame diffeomorphic image registration: Morphometric comparison of human and chimpanzee cortex. MIA (in press)

14. Parker, G.J.M., Alexander, D.C.: Probabilistic monte-carlo based mapping of cerebral connections utilising whole-brain crossing fibre information. In: Proc. IPMI. (2003) 684-695

15. Alexander, D.C., Barker, G.J., Arridge, S.R.: Detection and modeling of nongaussian apparent diffusion coefficient profiles in human brain data. MRM 48 (2002) 331-340

16. Cook, P.A., Alexander, D.C., Parker, G.J.M.: Modelling noise-induced fibreorientation error in diffusion-tensor MRI. In: Proc. ISBI. (2004) 332-336

17. Mardia, K.V., Jupp, P.E.: Directional Statistics. Wiley (2000)

18. Virgilio, G.D., Clarke, S.: Direct interhemispheric visual input to human speech areas. HMP 5 (1997) 347-354 